\title{
CD5 Positive Follicular Lymphomas- A Diagnostic Dilemma in a Resource Restricted Laboratory Setting
}

\author{
Sakthi Sankari $\mathrm{S}^{1 *}$, Arjunan $\mathrm{A}^{2}$, Bhuvaneswari M.G. ${ }^{2}$, Sindhuja $\mathbf{R}^{3}$, Rajeswari Thivya $\mathrm{D}^{4}$ \\ ${ }^{1}$ Department of Pathology, PSG Institute of Medical Sciences and Research, Coimbatore, Tamilnadu, India \\ ${ }^{2}$ Department of Pathology, Coimbatore Medical college, Coimbatore, Tamilnadu, India \\ ${ }^{3}$ Department of Pathology, Thirunelveli Medical college, Tamilnadu, India \\ ${ }^{4}$ Department of Pathology, Karpaga Vinayaga Medical College, Kanchipuram, Tamilnadu, India
}

\begin{abstract}
Background: Follicular lymphoma is an indolent B Non Hodgkin lymphoma which has a characteristic follicular growth pattern and immunohistochemical expression. The neoplastic cells express CD20, CD10, and BCL2 and are negative for CD5, CD23 and Cyclin D1. CD5 is a useful marker in the differentiation of various B cell lymphomas. Small cell lymphoma and mantle cell lymphoma express CD5. Some of the follicular lymphomas express CD5 which results in diagnostic confusion

Methods: 52 cases of lymphoma were diagnosed over a period of 2 years in a tertiary care centre in south India. Among these, 9 cases were diagnosed as follicular lymphoma based on the morphological and immunohistochemical study. A panel of antibodies was chosen based on the morphological diagnosis. The panel used for small cell lymphomas include CD20, CD3, CD5 CD23, Cyclin D1, CD10, BCL2 and Ki67.

Result: Of the nine cases of follicular lymphoma, three were CD5 positive. Two showed bone marrow involvement. There was no significant morphological difference between CD5 positive and CD5 negative follicular lymphoma.

Conclusion: The expression of CD5 by follicular lymphomas could be problematic when the follicular lymphoma as such lacks its characteristic follicular pattern andwhen there are no molecular studies available. Even though rare, follicular lymphoma is to be considered in the differential diagnosis of CD5 positive lymphomas.
\end{abstract}

Keywords: Follicular Lymphoma, CD 5 Positive, Indolent Lymphomas, B Cell NHL, Immunohistochemistry

\section{Introduction}

Follicular lymphoma (FL) is the second most common Non Hodgkin lymphoma (NHL) in India. It is an indolent lymphoma derived from germinal centre derived B cells. FL has a characteristic morphology with effaced nodal architecture, follicular growth pattern recapitulating germinal centres. Genetically, the hallmark of FL is $\mathrm{t}(14 ; 18) \mathrm{q}(32 ; \mathrm{q} 21)$ which has been detectable in a significant number of cases accounting to about $80 \%$. This genetic alteration is responsible for the deregulated expression of BCL2 which can be detected using immunohistochemistry. FL has characteristic immunophenotypic expression, positive for pan B cell markers, CD20, CD19, germinal centre associated antigens CD10, BCL6 and BCL2. FL do not express $\mathrm{CD} 5$, a $\mathrm{T}$ cell marker that has a key role in the differential diagnosis of small B cell Non Hodglkin lymphomas. ${ }^{[4-6]}$

CD5 is a glycoprotein expressed on mature $T$ cells and a small subset of B cells. CD5 positive cells in a lymph node are present in the follicular mantles. ${ }^{[7]}$ Among the lymphomas, CD5 is considered a marker for small lymphocytic (SLL) and mantle cell lymphoma (MCL). However a small subset of diffuse Large B cell lymphoma, Burkitt lymphoma and extranodal marginal zone lymphoma are found to have expressed CD5. ${ }^{[8,9]}$ And also, cases of CD5 positive follicular lymphomas have been reported in small numbers in the studies performed even before the advent of molecular studies. ${ }^{[10-12]}$

FL is basically a morphological diagnosis. Immunohistochemistry is used as an ancillary tool in the confirmation of diagnosis. CD5 expression however would result in diagnostic confusion when the morphology is not characteristic and flow cytometric and translocation studies cannot be done. In the present study, we describe morphologic and immunohistochemical features of 3 such cases of CD5 positive FL.

\section{Materials and Methods}

Fifty two cases of lymphoma were diagnosed over a period of 2 years in a tertiary care centre in south India. The lymphomas were reported based on the WHO classification of tumours (2008 classification). Among these, 9 cases were diagnosed as follicular lymphoma 
based on the morphological and immunohistochemical study. Histology was evaluated in formalin-fixed, paraffinembedded (FFPE) and H\&E-stained sections. Grading of FLs was based on the number of centroblasts per highpower field. A panel of antibodies were chosen based on the morphological diagnosis. The panel used for small cell lymphomas include CD20, CD3, CD5 CD23, Cyclin D1, CD10, BCL2 and Ki67.

For immunohistochemistry, $4 \mu$ sections were taken in specially coated slides. Slides were then deparaffinised and rehydrated in ethanol and xylene. Antigen retrieval was done by microwaving using two methods 1) by incubating sections with a Tris-EDTA solution, $\mathrm{pH} 9.0$ for 20 minutes before staining with antibodies against CD10, CD23, BCL2, CD5, CyclinD1, CD3 (mouse, monoclonal, biogenex), and 2) by incubating sections with $0.01 \mathrm{M}$ citrate buffer solution, $\mathrm{pH} 6.0$, for $20 \mathrm{~min}$ before staining with antibodies against CD45, CD20 (mouse, monoclonal, biogenex). The slides were then incubated under humid conditions at room temperature for 90 minutes.

The antibodies bound to antigens were detected by addition of secondary antibody conjugated with horse radish peroxidase polymer and diaminobenzidene substrate. The slides were counterstained with hematoxylin. Appropriate positive controls for the primary antibodies as per the manufacturer's instruction manual (biogenex) were used. The immunohistochemically stained slides were analyzed for the presence/absence of reaction and cellular localization. The positive reaction in the neoplastic cells was assessed. This study was conducted with approval of the Institutional ethical committee, in accordance with the ICMR guidelines for human research.

\section{Result}

Of the nine cases of FL, 3 were CD5 positive. All the three patients were males with the age range of 37 to 53 presenting with lymphadenopathy.

Histologically, all the three cases showed predominant follicular growth pattern, while one of them showed diffuse component accounting to less than $50 \%$.( fig 1 and 2). Histological grading was based on the number of centroblasts per high power field. On immunohistochemical study the cells were found to express CD20, CD10 and BCL2. (Fig 3 and 4) BCL2 was expressed in the follicular centres. Approximately $70 \%$ of the cells were CD5 positive. CD3 immunostaining was included in the panel to assess the proportion of $\mathrm{T}$ cells in follicles. As the $\mathrm{T}$ cells in the follicular centre usually express BCL2, CD3 immunostaining was compared with BCL2 before interpreting BCL2 expression in order to avoid misinterpretation. The follicular centre also had CD3 positive $\mathrm{T}$ cells which also expressed CD5 (fig5). Cyclin D1 was negative in all the three cases ruling out mantle cell lymphoma. (Table 1)

In case 2 there was weak expression of CD20 and CD5. Bone marrow trephine biopsies were subsequently received for these three cases. Two of the three cases showed bone marrow involvement, one was paratrabecular while the other was diffuse. There was no peripheral blood involvement. Extranodal involvement was not detected in any of the three cases.

The other six cases which have not been described here showed typical histological and immunohistochemical features of lymphoma.

\section{Discussion}

Follicular lymphoma is a mature B cell lymphoma which accounts for $20 \%$ of NHL.It is usually a disease of adults having a slight female preponderance. FL predominantly involves the lymph nodes, involvement of bone marrow and other extranodal sites can happen in the setting of widespread disease. Primary Extranodal FL can also occur. ${ }^{[1]}$

FL is a heterogenous neoplasm having a variable immunoarchitectural pattern. Typically the involved lymph nodes show complete effacement of nodal architecture by uniform sized follicles composed of centrocytes and centroblasts. These neoplastic follicles lack polarization and evidence of apoptotic activity (tingible body macrophages). The growth pattern can be follicular, follicular and diffuse and predominantly diffuse. CD23 immunostaining which highlights the follicular dendritic cells aids in assessing the extent of follicular component. ${ }^{[13,14]}$

The varied architectural patterns and variants lead to confusion with other small B cell lymphomas which include small lymphocytic lymphoma (SLL) and marginal

Table 1: Immunohistochemical feature of Follicular Lymphoma.

\begin{tabular}{|l|c|c|c|c|c|c|}
\hline & Histology & CD20 & CD10 & CD23 & CD5 & BCL2 \\
\hline 1. & Follicular & + & + & + in FDC & $+(70 \%$ of cells $)$ & + \\
\hline 2. & Diffuse $>50 \%$ & + (weak $)$ & + & - & $+(70 \%$ of cells $)$ & + \\
\hline 3. & Follicular & + & + & + in FDC & $+(50 \%$ of cells $)$ & + \\
\hline
\end{tabular}

FDC-follicular dendritic cell. 


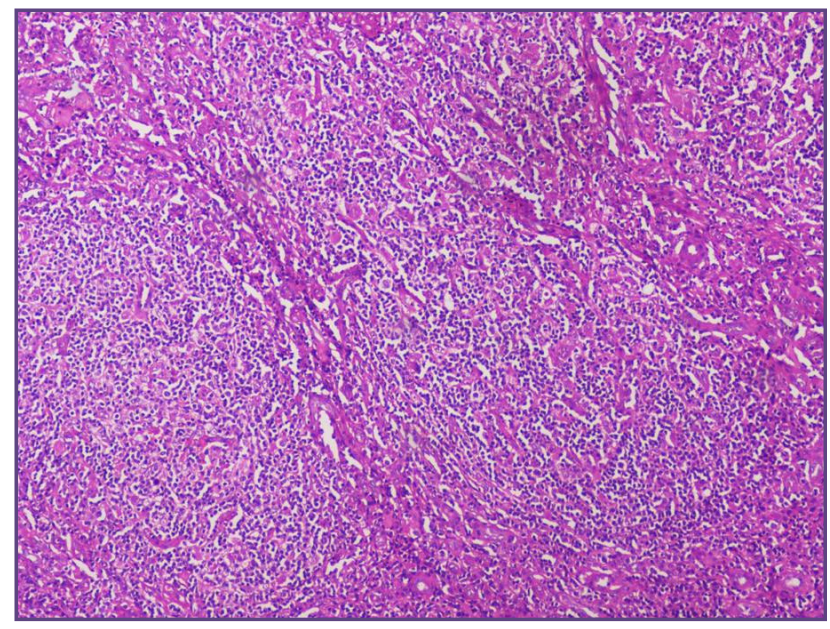

Fig. 1: Follicular growth pattern H\& E, 10X magnification.

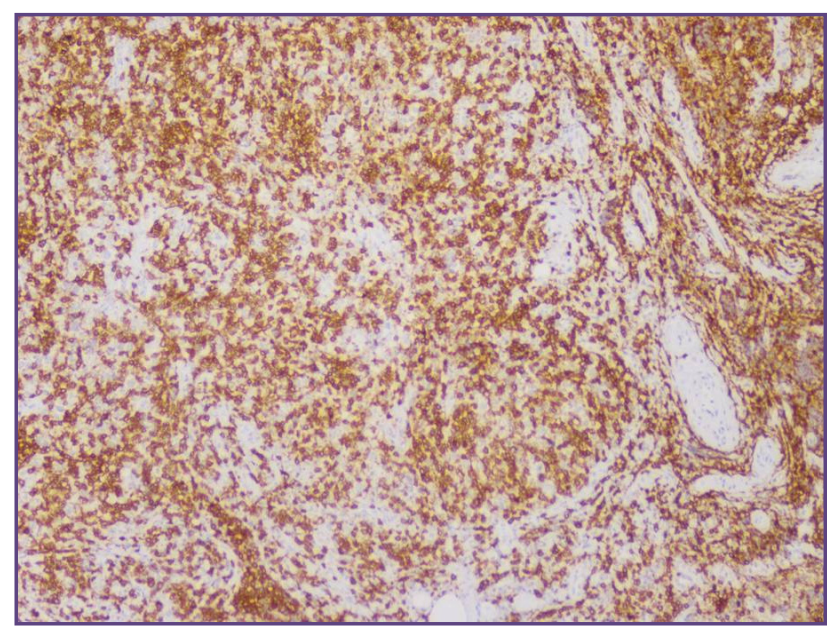

Fig. 3: Follicles expressing CD10 (IHC), 10X magnifiction.

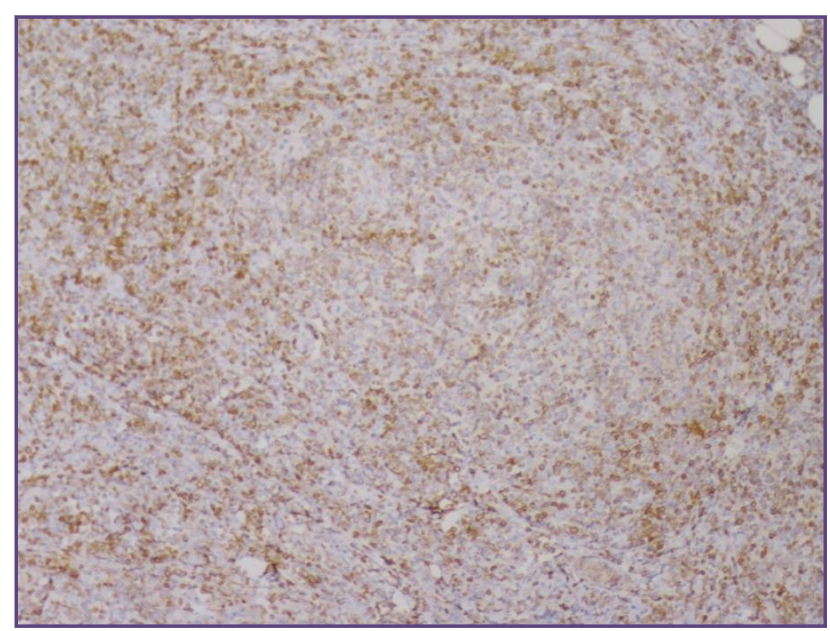

Fig. 5: Neoplastic cells expressing magnification.

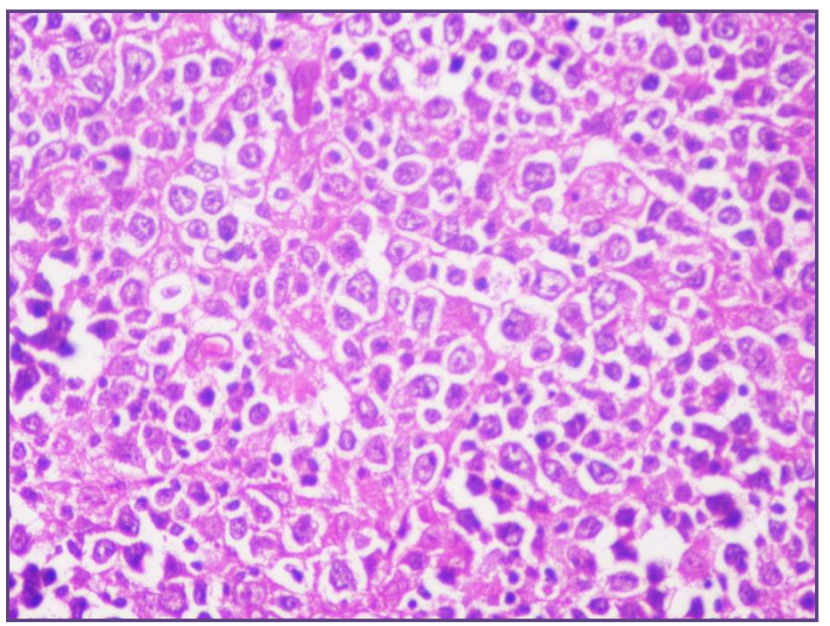

Fig. 2: showing follicular centres H\&E, 40X magnification.

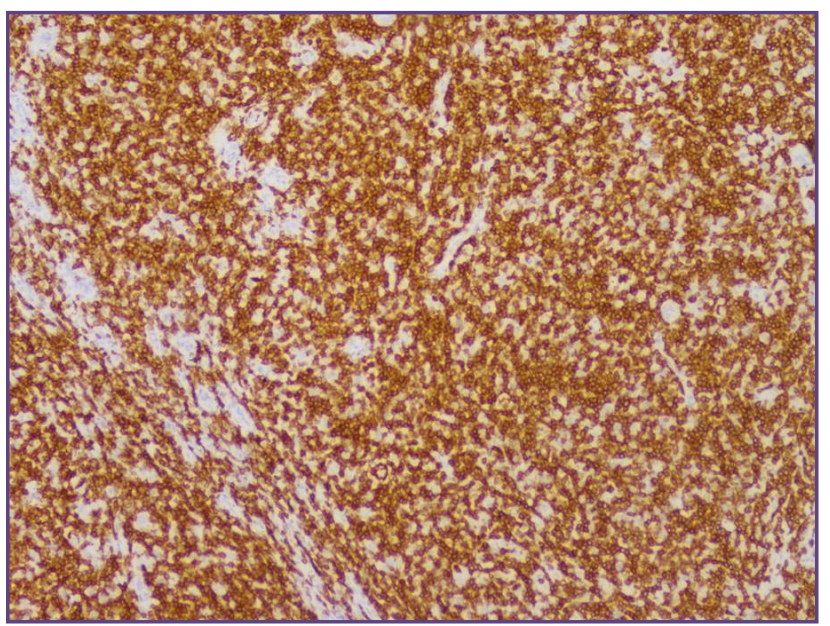

Fig. 4: Follicles expressing BCL2 (IHC), 10X magnification.

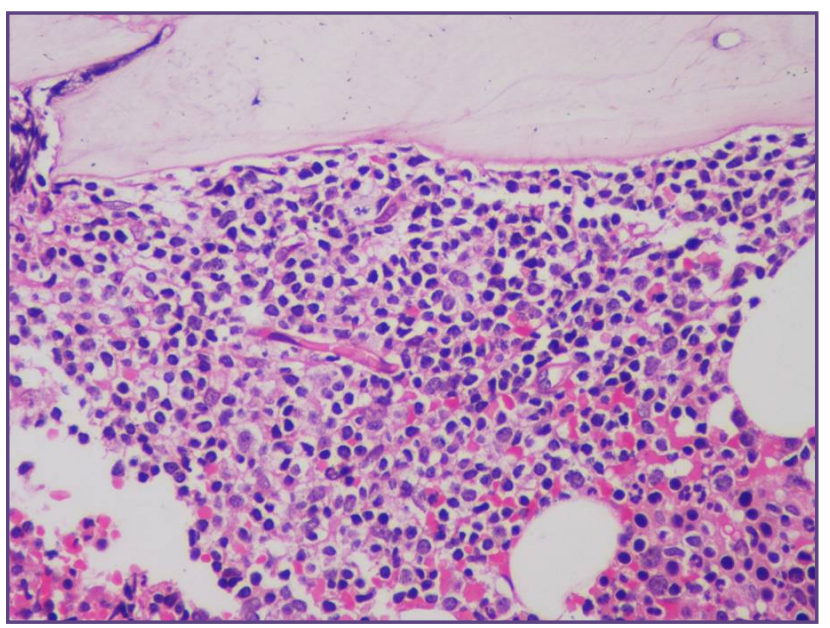

Fig. 6: Bone marrow trephine showing paratrabecular infiltrate of lymphoid cells.10X magnification. 
zone lymphoma. The typical immunoprofile of FL includes expression of CD10, BCL6, BCL2 and CD20. The expression of these markers are stronger in the follicles when compared to the interfollicular cells. ${ }^{[15]}$

CD10 is a germinal centre marker expressed both in reactive and neoplastic follicles. IT is also expressed in Burkitt and Diffuse Large B cell lymphoma. ${ }^{[15]} \mathrm{BCL} 2$ is an antiapoptotic protein which is negative in reactive germinal centres and overexpressed in FL, the expression of which is strong in grade 1 and $2 \mathrm{FL}$. It is a reliable marker for differentiating follicular lymphoma from follicular hyperplasia. $\mathrm{T}$ cells present within the follicular centres also express BCL2. ${ }^{[13]}$

CD5 is involved in T- and B cell receptor signaling. CD5 is a valuable marker in the differentiation of non Hodgkin B cell lymphomas. However CD5 is a mature T cell marker, but is expressed in a subset of B cell lymphomas (SLL and MCL). CD5 along with CD23 helps in the differentiation of small lymphocytic and mantle cell lymphoma. It has also been found to be expressed in marginal zone and diffuse large B cell lymphoma. ${ }^{[1-6]} \mathrm{CD} 5$ is one of the negative markers for follicular lymphoma. But CD5 positive follicular lymphomas have been reported to be a rare phenomenon. In the present study, 3 out of 9 cases of FL were CD5 positive, which appears more frequent when compared to the previous published studies.

They have been found to be associated with younger age, male predominance and grade 3 morphology. ${ }^{[15,16]}$ It has also been found to be expressed in follow up biopsies of FL which initially did not express.

In the present study, the age ranged from 37 to 53 . All the three cases were males. Morphologically, all the three cases had follicular growth pattern except for one which showed a predominant diffuse pattern. Two of the cases were grade 2, while the other was grade 3. CD5 positive FL has been reported to be associated with advanced disease and Extranodal involvement. One of the grade 2 and the grade 3 case showed bone marrow involvement. Bone marrow involvement was paratrabecular in grade $2 \mathrm{FL}$ (figure 6) and diffuse in grade 3 case. None of them had any evidence of Extranodal disease at presentation.

In the case 2 there was $>50 \%$ diffuse component and immunohistochemically, there was weak expression of CD20. This weak expression of CD20 is characteristic of small lymphocytic lymphoma. This weak expression of CD20 along with CD5 positivity made SLL a strong possibility. However CD23 was negative in that case.
Hence it was diagnosed as follicular lymphoma. CD5 positive follicular lymphomas reported so far has been confirmed with translocation and flow cytometric studies. These investigations were not conducted in the present study.

All the three cases showed morphological features of classical FL. No specific variants were found to comment on the association between $\mathrm{CD} 5$ positivity and any particular variant subtype. The other six cases of FL had wide age range (13 to 68), varied morphology, (4 of them grade 2 and the remaining two cases corresponding to grade 3 ).

The final diagnosis obtained was based on morphological and immunohistochemical study. Flow cytometry and cytogenetics were not performed to identify the translocation $\mathrm{t}(14,18)$ characteristic of FL. Follow up data was not available to understand the prognostic significance of CD5 expression in a follicular lymphoma.

\section{Conclusion}

CD5 positive FL can pose a diagnostic problem especially when the morphology is not characteristic including the presence of diffuse component and when genetic studies are not available. However the use of panel of markers can aid in the diagnosis.

In summary CD5 positive Follicular lymphomas had the same morphological and immunophenotypic expression as that of CD5 negative FL. Eventhough the frequency is very low, we need to consider the possibility of follicular lymphoma in the evaluation of CD5 positive B NHL.

\section{Reference}

1. Harris NL, Jaffe ES, Pileri SA, Stein H, Thiele J,Vardiman JW (eds). WHO Classification of Tumours of Haematopoietic and Lymphoid Tissues. IARC: Lyon, 2008; 220-226.

2. Horsman DE, Gascoyne RD, Coupland RW et al. Comparison of cytogenetic analysis, southern analysis,and polymerase chain reaction for the detection of $t(14 ; 18)$ in follicular lymphoma. Am J Clin Pathol 1995; 103:472-478

3. Stamatopoulos K, Kosmas C, Belessi C, et al. Molecular insights into the immunopathogenesis of follicular lymphoma. Immunol Today. 2000; 21:298-305.

4. Dogan A, Bagdi E, Munson P, et al. CD10 and bcl-6 expression in paraffin sections of normal lymphoid tissue and B-cell lymphomas. Am J Surg Pathol. 2000; 24:846-852.

5. Dorfman DM, Shahsafaei A. Usefulness of a new CD5 antibody for the diagnosis of T-cell and B-cell lymphoproliferative disorders in paraffin sections. Mod Pathol. 1997; 10:859-863.

6. Yin CC, Lin P, Carney DA et al. Chronic lymphocytic leukemia/small lymphocytic lymphoma associated with IgM paraprotein. Am J Clin Pathol. 2005; 123: 594-602. 
7. Leon ED, Alkan S, Huang JC, et al. Usefulness of an immunohistochemical panel in paraffin-embedded tissues for the differentiation of B-cell non-Hodgkin's lymphomas of small lymphocytes. Mod Pathol. 1998; 11:1046-1051.

8. Sundeen JT, Longo DL, Jaffe ES. CD5 expression in B-cell small lymphocytic malignancies: correlations with clinical presentation and sites of disease. Am J Surg Pathol. 1992; $16: 130-137$.

9. Dong HY, Gorczyca W, Liu Z et al. B-cell lymphomas with coexpression of CD5 and CD10. Am J Clin Pathol. 2003; 119:218-230.

10. Sekiguchi Y, Imai H, Wakabayashi M et al. CD5- positive follicular lymphoma: a case report and literature review. Intern Med. 2011; 50:899-904.

11. Mayson E, Saverimuttu J, Cartwright K. CD5-positive follicular lymphoma: prognostic significance of this aberrant marker? Intern Med J. 2014; 44:417-422.
12. Takata K, Miyata-Takata T, Sato Y, Yoshino T. Pathology of Follicular Lymphoma. J Clin Exp Hematop. 2014; 54(1): 3-9.

13. Fouad-Younes S, Beck A, Lossos IS, et al. Immunoarchitectural Patterns in Follicular Lymphoma: Efficacy of HGAL and LMO2 in the Detection of the Interfollicular and Diffuse Components. Am J Surg Pathol. 2010; 34(9): 1266-76.

14. Rizzo K, Nassiri M. DiagnosticWorkup of Small B cell Lymphomas: A Laboratory Perspective. Hindawi Publishing Corporation Lymphoma. 2012;

15. Barry TS, Jaffe ES. CD5+ Follicular Lymphoma A Clinicopathologic Study of Three Cases. Am J Clin Pathol 2002; 118:589-598.

16. Yu Li, Shimin Hu, Zhuang Zuo, Ming Hong. CD5-positive follicular lymphoma: clinicopathologic correlations and outcome in 88 cases. Modern Pathology 2015;28, 787-798.

*Corresponding author:

Sakthi Sankari S, Assistant Professor, Department of Pathology, PSG Institute of Medical Sciences and Research, Coimbatore, Tamilnadu, India, 641004, Phone: +91 04222570170

Email: sakthissankari@gmail.com

Date of Submission : 16.02.2017

Date of Acceptance : 10.06.2017

Financial or other Competing Interests: None.

Date of Publication : 11.12.2017 